\title{
Lattice Gauge Theory for a Quantum Computer
}

\author{
Richard C. Brower* \\ Boston University, Boston, MA 02215, USA \\ email: browerabu.edu
}

\section{David Berenstein}

University of California Santa Barbara, CA 93106, USA

email: dberensephysics.ucsb.edu

\section{Hiroki Kawai}

Boston University, Boston, MA 02215, USA

email: hirokikabu.edu

The quantum link [1] Hamiltonian was introduced two decades ago as an alternative to Wilson's Euclidean lattice QCD with gauge fields represented by bi-linear fermion/anti-fermion operators. When generalized this new microscopic representation of lattice field theories is referred as D-theory[2]. Recast as a Hamiltonian in Minkowski space for real time evolution, D-theory leads naturally to quantum Qubit algorithms. Here to explore digital quantum computing for gauge theories, the simplest example of U(1) compact QED on triangular lattice is defined and gauge invariant kernels for the Suzuki-Trotter expansions are expressed as Qubit circuits capable of being tested on the IMB-Q and other existing Noisy Intermediate Scale Quantum (NISQ) hardware. This is a modest step in exploring the quantum complexity of D-theory to guide future applications to high energy physics and condensed matter quantum field theories.

37th International Symposium on Lattice Field Theory - Lattice2019

16-22 June 2019

Wuhan, China

${ }^{*}$ Speaker. 


\section{Introduction}

Quantum Chromodynamics (QCD) remains a singularly difficult computational problem. In spite of the success of Wilson's Euclidean lattice QCD, there remains the notorious sign problem making predictions of real-time dynamics impossible, as well as severely limiting the determination of parton distribution functions and thermodynamics at non-zero chemical potentials needed by HEP experiments. On quantum computers, these sign problems are solved in principle. One defines a Hamiltonian operator for unitary evolution in continuous time. Two discretizations are required for any finite Qubit algorithm: i) Placing the Kogut Susskind Hamiltonian [3] on a finite volume lattice and replacing Euclidean time (it) by Minkowski time $(t)$ to guarantee unitary real time quantum evolution. ii.) The less familiar step of replacing field variables by a finite set of Qubits per lattice cell. The quantum link representation of Brower, Chandrasekar and Wiese [1] or D-theory accomplishes this second, field space quantization.

Since the introduction of D-Theory in the context of classical computing algorithms, it has continued to be developed for quantum computing of growing number of interesting theories reviewed by Uwe Wiese [4]. This talk will briefly present D-theory for QCD and then consider a simple prototype example of $U(1)$ gauge theory on a 2 d triangular lattice, which is capable of testing small kernel consistent with the hardware constraints in the NISQ era.

\section{Quantum link D-theory for QCD}

In going from the Euclidean D-theory to the Minkowski Hamiltonian, what is required, roughly speaking, is swapping the extra-dimension fermion with a gauge-fixed temporal realtime axis. The result is a wide range of spin and gauge field theories represented in the terminology of BravyiKitaev [5] as Fermionic Quantum Computing algorithms. For example, for QCD the Hamiltonian is

$$
\hat{H}=\frac{g^{2}}{2} \sum_{x, \mu} \operatorname{Tr}\left[\hat{E}_{L}^{2}(x, \mu)+\hat{E}_{R}^{2}(x, \mu)\right]-\frac{1}{2 g^{2}} \sum_{x, \mu, v} \operatorname{Tr}\left[\hat{U}_{\mu v}(x)\right]+\text { Quarks }
$$

The key to the construction is a faithful preservation of the symplectic gauge algebra by introducing on each link $(\langle x, x+\mu\rangle)$ operators,

$$
\begin{aligned}
& \hat{E}_{L}(x, \mu)=a_{\langle x, x+\mu\rangle}^{i \dagger} a_{\langle x, x+\mu\rangle}^{j} \quad, \quad \hat{E}_{R}(x, \mu)=b_{\langle x, x+\mu\rangle}^{i \dagger} b_{\langle x, x+\mu\rangle}^{j}, \\
& \hat{U}^{i j \dagger}(x, \mu)=\hat{a}_{\langle x, x+\mu\rangle}^{i \dagger} b_{\langle x, x+\mu\rangle}^{j} \quad, \quad U^{i j}(x, \mu)=b_{\langle x, x+\mu\rangle}^{i \dagger} a_{\langle x, x+\mu\rangle}^{j},
\end{aligned}
$$

expressed as bilinear of 6 color triplet and 6 anti-triplet fermionic operators with $i, j=1,2,3$. The full algebra of $E^{\prime} s$ and $U^{\prime} s$ are generators of $U(6)$. For QCD the unitary evolution, $\exp [ \pm i \hat{H}]$, in the Hilbert space was referred to as the QCD Abacus [6] illustrated in Fig. 1. The magnetic term acts on 3 color fermionic bits like beads on wires, changing the color flux on each link. The only deformation of the algebra is that on each link $\langle x, x+\mu\rangle$, the forward $(\hat{U}(x, \mu))$ and backward $\left(\hat{U}^{\dagger}(x, \mu)\right)$ gauge matrices no longer commute $\left(\left[\hat{U}, \hat{U}^{\dagger}\right] \neq 0\right)$ and they are paired with independent left $E_{L}(x, \mu)$ and right gauge $E_{R}(x, \mu)$. These no longer obey the identity $E_{R}=U^{\dagger} E_{L} U$. However since all link fields on two different links still commute, this algebraic deformation is an irrelevant UV cut-off effect typical of all lattice field theories. Extended Wilson paths at long 


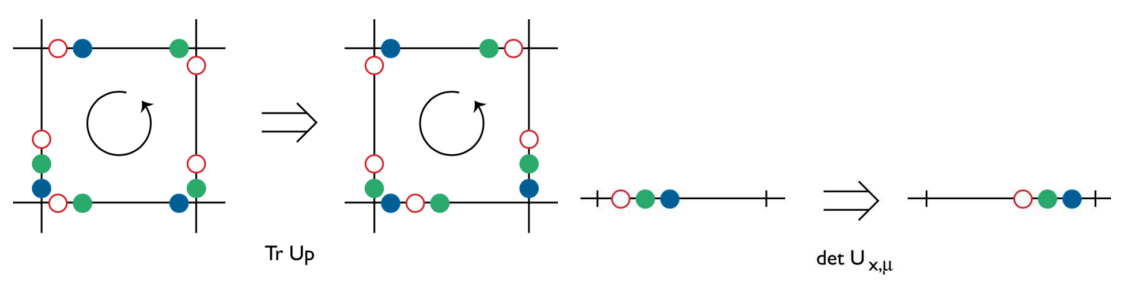

Figure 1: QCD quantum link dynamics: On the left, the Hamiltonian induces a hopping of link fermion to rotate various colors around a plaquette. On the right, by adding an extra determinant term the gauge group is broken from $\mathrm{U}(3)$ to $\mathrm{SU}(3)$ by translating a baryon-like object on the link.

distance will have only $O(a)$ commutators. D-theory conjectures [2] that this novel microstructure at the cut-off under proper implementation can provide a fundamental alternative to the Wilson Kogut-Suskind lattice gauge theories in the same universality class in the continuum limit. The precise conditions required to support this conjecture of course pose challenging theoretical and computational problems.

\section{Quantum Links for U(1) gauge Theory}

To test D-theory operation on existing Quantum Computing hardware, we now consider the simplest non-trivial confining gauge theory: the $2+1$ Abelian $U(1)$ theory on a triangular lattice. The compact $U(1)$ group manifold itself is a circle $S^{1}$, and the gauge algebra is realized as

$$
E_{L}=-E_{R}=-i \partial_{\theta} \quad, \quad U=\exp (i \theta)
$$

The single link compact manifold, $L^{2}\left(S^{1}\right)$, is infinite dimensional but it is a discrete set of Fourier modes in the flux basis, $|q\rangle$, enumerated by integer or half integer wave number $q$. In this Hilbert space we have that the action of $E_{L}, U$ is given by $E_{L}|q\rangle=q|q\rangle, \quad U|q\rangle=|q+1\rangle$. It is easy to check that $\left[E_{L}, U\right]=U$ and that this representation is irreducible. When we truncate the charge basis, $U$ and $U^{\dagger}$ needs to annihilate the largest and smallest charge states respectively.

D-theory replaces this manifold by the simplest truncation with a fermion bilinear representation, $\hat{U}=b^{\dagger} a$, on a two Qubit state. However since Fermion number, $\hat{N}=a^{\dagger} a+b^{\dagger} b$, is conserved on each link, we may project onto the two states with $\hat{N}=a^{\dagger} a+b^{\dagger} b=1$ and faithfully represent the D-theory algebra in terms of Pauli matrices,

$$
\hat{U}=\sigma^{+} \quad, \quad \hat{U}^{\dagger}=\sigma^{-} \quad, \quad \hat{E}_{L}=-\hat{E}_{R}=\sigma^{z}
$$

in a single Qubit space. Note in this Abelian example both left and right gauge generators at the ends of each link are the same up to a sign. Relative to the full $U(1)$ manifold, now the flux is restricted to $\pm 1 / 2$ on each link.

We need to extend this setup to allow for larger values of the flux. The angular momentum algebra can serve as a starting point for a representation of the equations $\left[E_{L}, U\right]=U,\left[E_{L}, U^{\dagger}\right]=$ $-U^{\dagger}$, with $E=L_{z}$ and $U \propto L^{+}$, so that the electric field squared is not just a constant like in the spin $1 / 2$ representation. We can get higher angular momentum representations by addition of 
angular momentum. This way we write the $E, U$ in terms of sums of spin $1 / 2$ representations. This decomposition by addition of angular momentum, allows us to define small kernels for existing NISQ hardware.

\section{Hamiltonian for $2+1 U(1)$ gauge theory}

In D-theory higher flux as we approach the continuum is built up by coherent states either in an extra dimension and/or at larger spatial distances. The dynamics of this coherence is an example of the typical physical mechanism requirement at a second order phase point in order to take the continuum limit. If achieved, universality requires a similar phase boundary familiar in the classical example of the Ising vs the $\phi^{4}$ Hamiltonian dynamics.

We consider the $2+1 \mathrm{~d} U(1)$ gauge theory Hamiltonian on a $2 \mathrm{~d}$ spatial lattice. Beginning with a single plaquette with $\pm 1 / 2$ quantum links we build up high flux by stacking them in an extra dimension and extending them on finite spatial volume. We being with the single spatial plaquette as the simplest toy model.

\subsection{Single Gauge Plaquette Model}

The Hamiltonian for a single triangular plaquette stacked up in an extra dimension enumerated by $s$ with periodic boundary conditions is

$$
\begin{aligned}
\hat{H}=\sum_{s}\left[\frac{g^{2}}{2} \sum_{j=1}^{3}\left(\sigma_{j, s}^{z}+\sigma_{j, s+1}^{z}\right)^{2}\right. & +\frac{\alpha}{2 g^{2}} \sum_{j=1}^{3}\left(\sigma_{j, s}^{+} \sigma_{j, s+1}^{-}+\sigma_{j, s}^{-} \sigma_{j, s+1}^{+}\right) \\
& \left.-\frac{1}{2 g^{2}}\left(\sigma_{1, s}^{+} \sigma_{2, s}^{+} \sigma_{3, s}^{+}+\sigma_{1, s}^{-} \sigma_{2, s}^{-} \sigma_{3, s}^{-}\right)\right] .
\end{aligned}
$$

The lattice structure of this spin-basis Hamiltonian is as in Fig. 2 with triangle links, each of which can be represented as one Qubit spatial link, stacked in the direction of the extra dimension. The

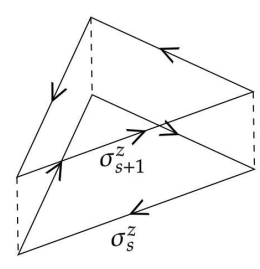

(a) Electric

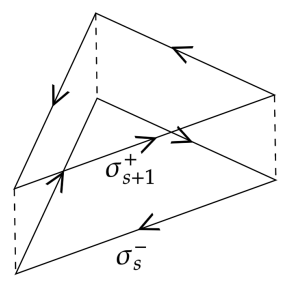

(b) XY Coupling

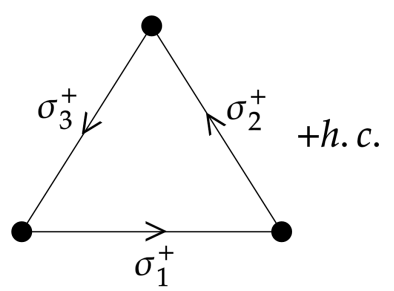

(c) Plaquette

Figure 2: Visualization of each term of the Hamiltonian of the single triangle plaquette model with two layers.

first term corresponds to the electric term $\hat{E}^{2}$ of (2.1), and third term to the plaquette term of (2.1) represents the magnetic $\hat{B}^{2}$ part of the energy. The positive and negative flux is created by $\sigma^{+}$ and $\sigma^{-}$respectively. Notice that on a single link $\hat{E}^{2}$ is constant, so we need at least one nearest neighbor to separate states based on differences of total electric flux. 
The second term can be rewritten as a sum of XX and YY interactions: $\sigma_{j, s}^{+} \sigma_{j, s+1}^{-}+\sigma_{j, s}^{-} \sigma_{j, s+1}^{+}=$ $\frac{1}{2}\left(\sigma_{j, s}^{x} \sigma_{j, s+1}^{x}+\sigma_{j, s}^{y} \sigma_{j, s+1}^{y}\right)$. It is an anti-ferromagnetic nearest neighbor XY spin $1 / 2$ chain for each link. Together with the first term, it composes a 1D spin-1/2 anisotropic XXZ model. There are no gauge variables on links into the extra direction so that the gauge theory still has only the proper $2 \mathrm{~d}$ spatial gauge rotations at each site. For example for a single $\triangle(1,2,3)$ plaquette model the generators are given by

$$
E_{i j}=\sum_{s}\left(\sigma_{i, s}^{z}-\sigma_{j, s}^{z}\right)
$$

where $(i, j)=(1,2),(2,3),(3,1)$. Given the periodic conditions and the commutation relations $\left[\sigma_{j}^{z}, \sigma_{i}^{ \pm}\right]= \pm \delta_{i j} \sigma^{ \pm}$, we may easily see that all three gauge operators satisfy $\left[E_{i j}, \hat{H}_{P}\right]=0$. The integer flux values for $E_{i j}$ at each site represent exact conserved Gauss' law sectors.

Now, we can expand this model to finite volume (areas in the two spatial dimensions) with multiple triangles for very small systems with periodic boundary conditions giving a $2 \mathrm{~d}$ torus. For example in Fig. 3 is a lattice of eight plaquettes triangulating a space for a $2 \times 2$ torus, but we may easily extend this to larger than $2 \times 2$. The triangle plaquette tiling forms a bipartite dual hexagonal lattice in Fig. 3(right), where the Pauli terms of the Hamiltonian associated with one fixed color commute with each other. This leads naturally to a term Trotter expansion where one can parallelize the evolution on a quantum computing algorithm with gauge invariant kernels on plaquettes. These are implemented next as quantum circuits for single 2 layer single plaquette model.
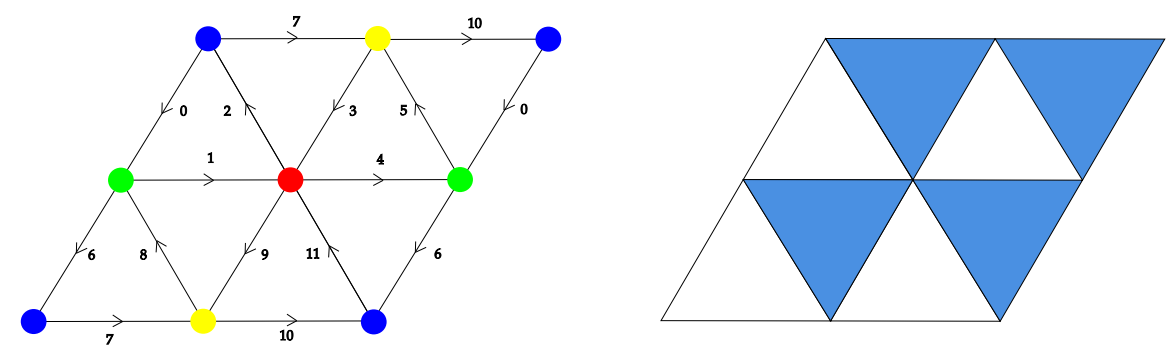

Figure 3: Example of triangulations of two dimensional spaces for $2 \times 2$ torus. Tessellation of $2 \times 2$ torus. On the left the lattice has 12 independent links (labeled with numbers), 4 independent vertices (labeled with colors) and 8 independent faces. With two layes this is 24 Qubit Hamiltonian On the right we color the lattice into two sets of face as a bipartite dual hexagonal lattice.

\subsection{Gauge Invariant Trotter Decomposition and Quantum Circuits}

In order to simulate the time evolution $e^{-i \hat{H} t}|\psi\rangle$ of the plaquette model on a digital quantum computer, the interactions between the links are represented as entanglements between the corresponding Qubits. Each of the exponentials of $\hat{E}^{2}$, coupling, and $\hat{B}^{2}$ terms can be represented as a quantum circuit as Fig. $4 \mathrm{a}, 4 \mathrm{~b}$, and $4 \mathrm{c}$ respectively. The coupling constant information and the evolution time $t$ in encoded in the rotation angle of the $R Z$ and $R X$ gates. The $X X$ and $Y Y$ terms commute with each other and together are gauge invariant. The other two are gauge invariant on their own. The separate non-commuting terms in $\hat{H}$ can be approximated via the Trotter decomposition exactly maintaining gauge invariance and unitarity. Introducing small time intervals 


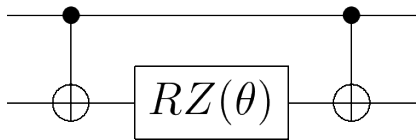

(a) Electric $\left(e^{-i \theta \sigma^{z} \otimes \sigma^{z}}\right)$

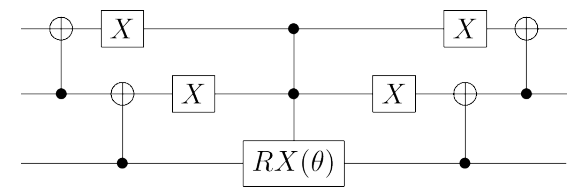

(c) Plaquette $\triangle$

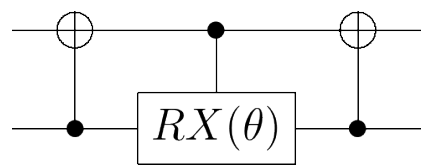

(b) $\mathrm{XY} \operatorname{term}\left(e^{-i \theta\left(\sigma^{+} \otimes \sigma^{-}+h . c .\right)}\right)$

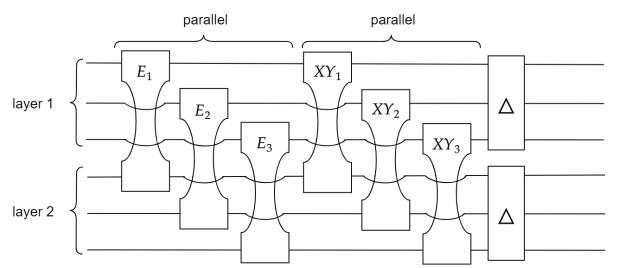

(d) Overall step

Figure 4: The quantum circuit representing each term of the Hamiltonian of the single plaquette model (a)-(c), and the circuit overview for the entire one single Trotter step (d).

$\Delta t=t / n$, the Trotter evolution for $n$ steps is

$$
e^{-i \hat{H} t}|\psi\rangle=\left(e^{-i \hat{H}_{E} \Delta t} e^{-i \frac{\alpha}{2 g^{2}} \sum_{j=1}^{3}\left(\sigma_{j, s}^{+} \sigma_{j, s+1}^{-}+\sigma_{j, s}^{-} \sigma_{j, s+1}^{+}\right) \Delta t} e^{-i \hat{H}_{B} \Delta t}\right)^{n}|\psi\rangle
$$

For the single plaquette model, one Trotter step is illustrated in Fig. 4d. Since different Qubit Pauli matrices commute with each other, the multiple terms for $\hat{E}^{2}, \hat{B}^{2}$, and the inter layer XY coupling may be implemented in parallel on a quantum circuit. Fig. 5 demonstrates an example of the time evolution for the single plaquette model Hamiltonian with Trotter decomposition with two different time intervals $\Delta t=0.5$ and $\Delta t=0.125$, compared with the exact computation.

For the multiple plaquette model, we may simply duplicate and repeat this kernel, adding only one additional layer for the two groups of the plaquette terms with different colors which do not commute with each other.

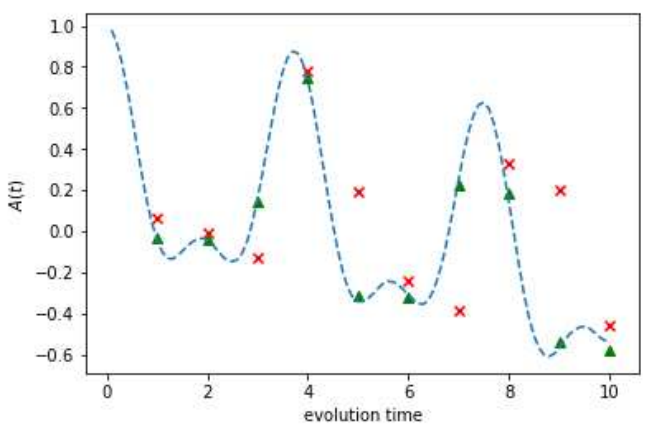

Figure 5: Persistence amplitude $A(t)=\left\langle\phi\left|e^{-i \hat{H} t}\right| \phi\right\rangle$ for a single triangle model with $g=0.75$ and $\alpha=1$ from $t=1$ to $t=10$. The state $|\phi\rangle$ is initialized in the $|+\rangle$ state for all Qubits. The blue dotted line represents the exact values of this inner product, the red and green points represent the Trotter result with a time interval $\Delta t=0.5$ and $\Delta t=0.125$, respectively. 


\section{Future Directions}

We have discussed a possible implementation of the $U(1)$ gauge theory in $2+1$ dimensions on a quantum computer made of Qubits. The small kernels identified in this paper are typical of kernels encountered in a variety of theories. Investigating alternative Qubit circuit implementations and testing them in early Noisy Intermediate-Scale Quantum (NISQ) platforms is useful. This is a process of both refining code kernels and evaluating quantum hardware systems.

D-theory for $d=4$ gauge and $d=2$ chiral spin theories with asymptotic freedom scaling have a clear path to the continuum due to the gapless phase in $d+1$. For the Abelian case, a plausible route to the coherent formation of large flux at physical scales is less well understood. Perhaps a promising avenue of investigation of this two state triangular lattice Abelian quantum link model is to look at its duality to a spin model on a hexagonal lattice following arguments similar to the classic duality of the $3 \mathrm{~d}$ of Ising model and Wegner's $3 \mathrm{~d} Z_{2}$ gauge theories on a square lattice.

Finally there are many interesting small quantum lattice prototypes. For example in addition to toroidal geometries with periodic boundary condition, one may consider spherical or de-Sitter lattices starting with the sequence of platonic solids. One can even consider small hyperbolic or Anti-de-Sitter lattices generated by the triangle group [7]. In conclusion even within the limited context of $U(1) / Z_{2}$ quantum links in the $2+1$ setups, there is a rich theory landscape for testing methods and confronting fundamental problems in quantum computing.

\section{Acknowledgements}

We are grateful to Cameron V. Cogburn for fruitful discussions. This work was supported in part by the U.S. Department of Energy (DOE) under Award No. DE-SC0019139.

\section{References}

[1] R. Brower, S. Chandrasekharan and U. J. Wiese, "QCD as a quantum link model," Phys. Rev. D 60, 094502 (1999) doi:10.1103/PhysRevD.60.094502 [hep-th/9704106].

[2] R. Brower, S. Chandrasekharan, S. Riederer and U. J. Wiese, "D theory: Field quantization by dimensional reduction of discrete variables," Nucl. Phys. B 693, 149 (2004) doi:10.1016/j.nuclphysb.2004.06.007 [hep-lat/0309182].

[3] J. B. Kogut and L. Susskind, "Hamiltonian Formulation of Wilson's Lattice Gauge Theories," Phys. Rev. D 11, 395 (1975). doi:10.1103/PhysRevD.11.395

[4] U. J. Wiese, “Towards Quantum Simulating QCD,” Nucl. Phys. A 931, 246 (2014) doi:10.1016/j.nuclphysa.2014.09.102 [arXiv:1409.7414 [hep-th]].

[5] Sergey Bravyi and Alexei Kitaev, "Fermionic quantum computation" in Annals of Physics, Vol. 298, Iss. 1 (2002) pp.210-226

[6] R. C Brower, "The QCD abacus: A New formulation for lattice gauge theories" in Recent developments in nonperturbative quantum field theory. Proceedings, APCTP-ICTP Joint International Conference, Seoul, Korea, May 26-30, 1997

[7] R. C. Brower, C. V. Cogburn, A. L. Fitzpatrick, D. Howarth and C. I. Tan, "Lattice Setup for Quantum Field Theory in $\mathrm{AdS}_{2}, "$ arXiv:1912.07606 [hep-th]. 